\title{
Roles in social interactions: graphlets in temporal networks applied to learning analytics
}

\author{
Raphaël Charbey ${ }^{1}$, Laurent Brisson ${ }^{1}$, Cécile Bothorel ${ }^{1}$, Philippe Ruffieux ${ }^{2}$, \\ Serge Garlatti ${ }^{1}$, Jean-Marie Gilliot ${ }^{1}$, and Antoine Mallégol ${ }^{1}$ \\ 1 IMT Atlantique, Lab-STICC UMR CNRS 6285, F-29238 Brest, France \\ firstname.name@imt-atlantique.fr \\ 2 Usages du numérique et Didactique de l'informatique (MUNDI), Av. des Bains 21, \\ CH 1014 Lausanne VD, Switzerland \\ http://www.sqily.com
}

\begin{abstract}
There is a growing interest in how data generated in learning platforms, especially the interaction data, can be used to improve teaching and learning. Social network analysis and machine learning methods take advantage of network topology to detect relational patterns and model interaction behaviors. Specifically, small induced subgraphs called graphlets, provide an efficient topological description of the way each node is embedded in the meso-scale structure of a network. Here we propose to detect the roles occupied by the different participants, students and teachers, in the successive phases of courses modeled by a sequence of static snapshots. The detected positions, obtained thanks to graphlet enumeration combined with a clustering method, reveal the different roles observed in each snapshot. We also track the role changes through the overall sequence of snapshots. We apply our method to the Sqily platform and describe the mutual skill validation process. The detected roles, the transitions between roles and a overall visualization through Sankey diagrams help interpreting the course dynamics. We found that some roles act like necessary steps to engage students within an active exchange process with their classmates.
\end{abstract}

Keywords: temporal networks, social interactions, motifs, graphlets, learning analytics, role detection

\section{Introduction}

Networks provide a framework for studying complex systems in a broad set of fields, ranging from biology, telecommunication to social networks and e-learning. Actors and their relationships are modeled as graphs, where edges represent friendship or any kind of interaction. For example in the field of e-learning, as it is now well accepted that a student's collaboration [7] is central for facilitating the learning process, learning platforms propose discussion forums and social networking facilities [6], and teachers are encouraged to design pedagogical activities involving interactions between learners. Social Learning Analytics [9] denotes a growing interest in managing interaction data, in order to improve 
teaching and learning, to assist educational institutions in increasing student retention and improving student success.

Network properties such as density, centrality, and degrees are very helpful for understanding the structure of a peer network, e.g. how students engage in learning and performance, both as individuals and as groups [22]. But detecting and interpreting patterns of exchanges that occur between the students is another step to understand the dynamics and identify or predict problematic situations. Understanding these patterns will help us understanding the course organisation and the role of each individual within the overall organisation.

The patterns of exchanges are likely to be detected through induced subgraph enumeration, that offers a competitive as well as an intuitive insight of the meso-scale structure of networks and allows for characterizing them relatively from one another. The induced subgraphs, also known a graphlets, also provide a native role detection support since nodes can be characterized by their positions in the graphlets. By studying the node roles in the successive phases of a temporal network, which can be modeled by a sequence of static graphs, one can understand their evolution and the way they transit from a role to another.

Contributions In this article, we provide a methodology for describing interaction networks in a dynamic way at node level. We first show how to find graphlets in static networks. We then consider the problem of role detection. The position of a node in a graphlet describes the place it occupies in its neighborhood. We use a graphlet-based node embedding method which explicitly counts its different positions occupied in interaction schemes involving 3 nodes. Then we provide a data-driven way to build interaction profiles as a combination of positions representing the different role specific to the considered network.

In temporal networks, nodes are involved in different graphlets, and therefore roles, as time goes by. We discretize time by converting temporal information on edges, i.e. the timestamp when the interactions occurred, into a sequence of static networks called snapshots. By assigning roles to nodes during each snapshot, our analytical approach explores how they move from one role to another. Finally we propose a longitudinal visualization, with Sankey diagrams, of the transitions from one role to another throughout the life of a temporal network.

We apply this methodology to e-learning data from the Sqily platform dedicated to the mutual validation of skills. We thus illustrate how interaction patterns (based on the validation of skills) can make it possible to associate roles with learner behaviors. Static and dynamic analyses of these roles thus provide students and teachers with useful insights.

\section{Related work}

Networks and learning analytics Social network analysis (SNA) has proven extremely powerful at describing and analysing network behaviors in e-learning. A systematic review of the literature on SNA in this field [4] covers more than 30 studies which analyze interaction patterns in forums and where centrality 
and density measures are mostly used. For example, in [26], low or high in- or out-degree centrality scores exhibit popular students who provide comments to others, who are reflectors and good communicators in the learning process or play knowledge broker's roles. Let us note that most of these studies deal with very small graphs (only a few dozen nodes).

The visualization of networks is an approach to explain the nature of community dynamics. KISSME is an example of an interactive visualization tool of content-aware interactions among learners [27]. Behavioral links (contributions such as "reply", "reference" and "annotate") can be overlaid on the learner-time display to show how patterns of interaction change over time.

Motifs in temporal networks While dealing with large complex networks, one might need automatic ways to count and detect relevant, sometimes non obvious, behaviors. This is included in the scope of application of subgraph pattern detection in networks $[12,20]$. In temporal networks, there is the constraint that edges have a timestamp, and the graphlets sought are induced subgraphs occurring in a wider network structure during specified time windows, with nodes interacting in an ordered sequence $[13,16]$. When looking for motifs, we are interested in how many times each pattern occurs. An efficient counting of temporal graphlets is proposed in [21], along with a framework to compare the structure of several complex systems, e.g. they show that email exchanges and Facebook wall posts do not involve the same motifs and therefore reveal different communication behaviors.

In this work we are not interested in the sequences of interactions and temporal motifs, but more in the static motifs we observe at different phases of a teaching. instantiated in the learning analytics field, our goal is to understand with whom learners interact, and the evolution of their position in the group at different times during a course.

Subgraph enumeration and nodes embedding If we discretize time and study interactions on successive snapshots, i.e. successive static graphs that are the result of an aggregation of interactions occurring during specified time windows $[1,8]$, the detection of motifs then consists in searching for static patterns within these snapshots. Hulovatyy et al. have introduced patterns that cover several consecutive snapshots and the persistence of edges through time [14]. Braha and Bar-Yam have enumerated the subgraphs of consecutive networks as if they were static ones [3] which is the strategy we adopt since the nodes we are dealing with are not always active in consecutive snapshots.

Graphlets are very useful to exhaustively list interaction patterns through, for example, a systematic enumeration of all possible subgraphs of a chosen size. A network may be characterized by a vector composed of the relative frequency of each graphlet, the ratio between its number of appearances and the total number of graphlets in the network [23]. Another possible vector is based on the graphlet representativity, that is the ratio between its relative frequency within a network and its relative frequency within a set of networks [5]. Moreover they allow for 
easy interpretation of the results. They also can be used as an embedding method to model nodes' neighborhood $[19,11]$. By enumerating the positions (or orbits) in which nodes appear, the graphlets offer a way to compare their topological similarity.

\section{Method}

We define a temporal oriented graph $G=(V, E)$ in which each edge $(u, v, t s)$ means that the user $u \in V$ interacts with the user $v \in V$ at time $t s$. We discretize time by converting temporal information into a sequence of $T$ snapshots. The interactions that occur during the period of each snapshot are aggregated leading to a set of $T$ graphs $\left\{G_{1}, \ldots, G_{T}\right\}$ where $G_{t}$ is the directed graph representing the interactions between active nodes that occurred within the $t$-th time slice.

The first step of our proposal is to enumerate the different possible graphlets of size $k$ and then search for them in the static graphs $G_{t}$. The second step is to compute position-based embeddings for the nodes in each $G_{t}$ relatively to their positions within the different graphlets. Finally, a clustering step over the nodes vectors produce complex interaction profiles, mixing positions and exhibiting roles as distributions of frequencies of positions in graphlets.

Size $k$ graphlet enumeration consists on visiting every possible combination of $k$ nodes such that the subgraph induced by these nodes (the nodes themselves and all edges between them) is connected. Each of these subgraphs is then identified as isomorphic to one of the graphlets and the relative enumeration counter is incremented. The list of size-3 directed graphlets is presented in Figure 1.

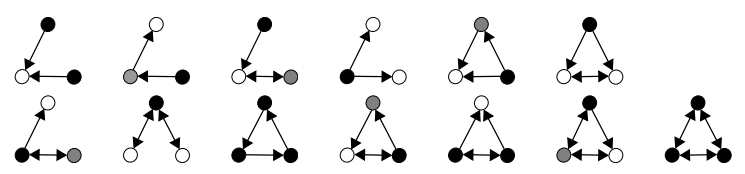

Fig. 1. The 13 directed graphlets of size three and their respective positions depicted by shades of grey. In each graphlet, the nodes with the same colour are in the same position.

Every position is interesting to observe. Intuitively, appearing in the central $\therefore$ or peripheral $\dot{C}$ orbit of the star does not correspond to the same role, even though the graphlet is the same.

As our goal is to describe the different positions that nodes occupy over time, and in order to build snapshots that will lead to the role dynamics, we focus on the temporal separation between two events as a referee for time discretization. We consider that a time window $\delta$ without any interaction is the marker of a break in the network dynamics which would result in an other organization. Each snapshot $G_{t}$ therefore corresponds to a sequence of events so that two consecutive events are separated with less than $\delta$. 
On each snapshot, we enumerate the positions of each of their nodes using Fanmod process [28]. Then comes the step of nodes embedding. For each node in each snapshot, we compute the frequency of each of its positions as follows: the frequency of a vertex $v$ in a position $p$ is the ratio of the number of appearances of $v$ in $p$ with the total number of appearances of $v$ in any position. This embedding is an adaptation of a popular graphlet-based network embedding [23] to the position level.

The set of position-based vectors are the exhaustive list of the different distributions of positions held by nodes, compiling the exhaustive set of behaviors detected at the different periods of the lifetime of the current temporal network. By applying the kMeans algorithm $[17,18]$ to these vectors, we obtain clusters of similar distributions of positions. The clustering produced by this algorithm is dependent on its initialization step and the number of clusters $k$ that is given as a parameter. Therefore we run the algorithm a hundred times for each value of $k$ between 1 and 20 and kept the best result in term of the silhouette score [24].

The resulting clusters define sets of nodes whose embeddings are similar. In our mutual validation course case-study, a user's embedding describes how $\mathrm{s} /$ he collaborate with others, by validating or being validated, and by whom. Through the experiment detailed in the next section, we will illustrate the power of expressiveness of positions in graphlets.

\section{Case study from the Sqily platform}

This case study focuses on interactions on the Sqily ${ }^{3}$ learning platform, whose particularity is to promote the mutual validation of skills. We first show how we constructed a dataset from the available information on the mutual validation of skills, then we present the topological roles extracted by our method by linking them to the corresponding student behaviors. Finally, we conduct a longitudinal study that highlights the value of these roles in exploring the dynamics of interactions.

\subsection{Mutual validation of skills}

In this context we use the term skills in the broad sense, i.e. knowledge, capacities, know-how and professional skills. The principle of mutual validation of skills is to encourage the learner to adopt a reflective approach: $\mathrm{s} /$ he must mobilize her/his newly acquired skills in order to explain them and help other students acquiring them. The learner thus puts herself/himself in the role traditionally assigned to the teacher and deepens her/his skills.

The mutual validation of skills has two inspirations: mutual teaching [10] (verbalizing knowledge, exchanging with peers, improving understanding through repetition) and knowledge trees [2] (graphic representation of competencies,

\footnotetext{
3 http://www.sqily.com
} 
knowledge management, valuing expertise). According to these principles, the Sqily platform:

- allows to define a tree of skills,

- allows students who master a skill to create assessments and validate skills of others students.

\subsection{Dataset description}

In this case study we analyze the behavior of students and teachers in terms of skills validation. Each course is modeled as a graph $G$; edges $(u, v, t s)$ represent mutual skill validations where the user $u$ validates one skill of the user $v$ at time ts.

Our dataset is comprised of 11 courses that have an average of 58 students. There is a strong involvement in mutual validation: $30 \%$ of the skills were created by students and $70 \%$ of the evaluations were done through peer-reviewing. However, students validated only $40 \%$ of the skills for which they started the activity sequence.

We arbitrarily set the size of the separating window between two snapshots at 15 days (parameter $\delta$ ). It generated a total of 64 graphs distributed differently over the courses: they are divided in at least 1 and up to 19 phases, with an average of 3.6 phases. The phases last at least 1 day and up to 103 days, with a mean of 32 days. The snapshots have an average of 21 vertices and 60 edges but they are also very heterogeneous with a minimum of 3 vertices and 2 edges and a maximum of 102 vertices and 370 edges.

\subsection{Role characterization}

The topological framework based on position enumeration led us to obtain roles, which positions frequencies are summarized in Table 1. We describe these roles by characterizing them with variables that were not used to define them: the proportion of teachers/students (see Table 2), the student peer-reviewed rate (average rate of evaluations made by peer students) and the commitment level (rank of skill acquisition in the course) (see Table 2). We present the roles obtained in two sets: the first includes the roles most involved in validating the skills of other learners, while the second includes roles concerned about getting validated.

\section{Assessor oriented roles}

- Expert The Expert has mastered a skill and validates a large number of other learners. These learners generally are validated by the Expert alone. This role mainly brings together teachers and learners who will embody the role of the teacher in one or more skills. It mainly corresponds to an assessor's behavior: $87 \%$ of its positions are the source of the edges. Moreover, they are those with the lowest skill acquisition rank, which highlights their early involvement in courses. 
Table 1. Roles description: Frequencies of positions within graphlets (multiplied per 100). The four left positions reflect active validation, the 3 positions in the middle of the table are intermediate ones, whereas the 4 ones on the right of the table represent passive postures in the validation process. The roles are also classified verticaly, depending on their ratio of teachers.

\begin{tabular}{|c|c|c|c|c|c|c|c|c|c|c|}
\hline & \multicolumn{4}{|c|}{ Assessor's positions } & \multicolumn{2}{|c|}{ Mixed positions } & \multicolumn{4}{|c|}{ Assessee's positions } \\
\hline & $\Leftrightarrow$ & $f$ & & 6 & $\int_{0}$ & $\leftrightarrow \leftrightarrow_{0}$ & $\sum_{i \infty}^{\infty}$ & $\&_{0}$ & $P_{0}$ & 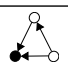 \\
\hline Peer Assessed Learner (Late) & & & & 1 & 2 & & 27 & 54 & 11 & 1 \\
\hline Expert Assessed Learner & & & & 1 & 1 & & 91 & 4 & 1 & 1 \\
\hline Peer Assessed Learner & 1 & 2 & & 4 & 5 & 1 & 60 & 16 & 7 & 2 \\
\hline Proxy Teacher & 17 & 19 & 4 & 54 & 2 & & & & & \\
\hline Committed Learner & 18 & 13 & 1 & 11 & 14 & 2 & 22 & 5 & 3 & 1 \\
\hline Expert & 64 & 11 & 5 & 7 & 5 & 1 & 3 & 1 & & \\
\hline
\end{tabular}

Table 2. The left side of the table presents the distribution of roles among all courses according to student or teacher status. The right side characterizes them upon their commitment to the mutual validation of skills. Student peer-reviewed rate is the average rate of evaluations made by students. Skill acquisition rank orders the learners of a course in a normalized way (between 0 and 1 ).

\begin{tabular}{l|cc||cc} 
& \multicolumn{3}{|c||}{ Students Teachers } & Student-reviewed Skill acquisition \\
rate (mean) & rank (median) \\
\hline Expert & 14 & 40 & 0.46 & 0.27 \\
Proxy Teacher & 44 & 3 & 0 & 0.45 \\
Committed Learner & 146 & 19 & 0.75 & 0.40 \\
Expert Assessed Learner & 239 & 1 & 0.34 & 0.43 \\
Peer Assessed Learner & 248 & 4 & 0.79 & 0.53 \\
Peer Assessed Learner (Late) & 39 & 0 & 0.94 & 0.74 \\
\hline
\end{tabular}

- Proxy Teacher While holding the Proxy Teacher role, a user (mostly a student) focuses exclusively on validating the skills of her/his peers. The positions are concentrated on assessor's ones, with a majority of co-validation with another assessor (the Experts are conversely the only assessor most of the times). While being themselves validated, the users in this role never ask for peer-validation, which is consistent with their early involvement in the courses (like the Experts).

- Committed Learner The Committed Learner is balanced in her/his approach to mutual validation of competence: s/he quickly validates her/his skills (0.40 skill acquisition rank) and adopts a reflective approach over the same period by validating other learners. It is the second role with the highest number of teachers but also one of the most demanding in terms of student-review. 


\section{Assessee oriented roles}

- Expert Assessed Learner The Expert Assessed Learner is focused on getting assessed, and relies almost exclusively on an Expert. Indeed, 91\% of their positions are in the target of the star $\&$ and they have a low student-reviewed rate (0.34). This role is played by students (all occurrences but one) who obtain their skills rather quickly (0.43 skill acquisition rank) which may indicate that they have had no choice but to ask their teacher for validation.

- Peer Assessed Learner The Peer Assessed Learner is mainly focused on receiving validation of her/his skills and relies mainly on students to do so. $\mathrm{S} / \mathrm{He}$ 's mostly validated within the star pattern $\dot{\ell}_{\bullet}$, but also appears in $\therefore$ and $\leftarrow$. This role is played by students (248 over 252$)$ who acquire their skills more slowly (0.53 skill acquisition rank) than Expert Assessed Learners.

- Peer Assessed Learner (Late) This role includes a sub-category of Peer Assessed Learners who validate their skills later relatively to others. Their positions are distributed among the assessee's positions with a high rate of $\therefore$ (transitively validated). These students are the last ones to validate skills (0.74 skill acquisition rank), which allows their validators (94\% of students) to validate some skills before proposing their own assessments.

This topological description, combined with behavioral indicators, highlights the variety of roles taken by students and teachers throughout a course. These roles make it possible to suggest several hypotheses about learners' behavior. To do so, it is interesting to investigate the changes in roles during the different phases of the course. Therefore we provide a longitudinal study through a Sankey diagram, a visualization tool allowing to describe node flows between different steps [15].

\subsection{Examples of a course's dynamics}

Figure 2 depicts the evolution of roles (each color line) within the phases (each vertical line) of one of the courses. It is interesting to note that the course begins with a first phase where the teacher, in Expert role, validates skills to some Expert Assessed Learners. In a second phase, more students arrive. They occupy all different roles and many Expert Assessed Learners from the first phase are now acting as Experts and Committed Learners.

We also may focus on some specific phases, for instance phases 9 et 10, whose networks $G_{9}$ and $G_{10}$ are showed in Figure 2. In phase 9, that lasts 10 days, a student, user 54 , is acting as an Expert, i.e. as a source of many stars $\underset{C}{\rightarrow}$, since he or she is the only assessor of several other students. The teacher, user 5 , appears as a Committed Learner since s/he shares a lot of co-assessor's positions C. (all the students s/he validated are co-validated by other users) and in the first position of the transitivity subgraph $\ell$. with user 54 next to him $\&$. 

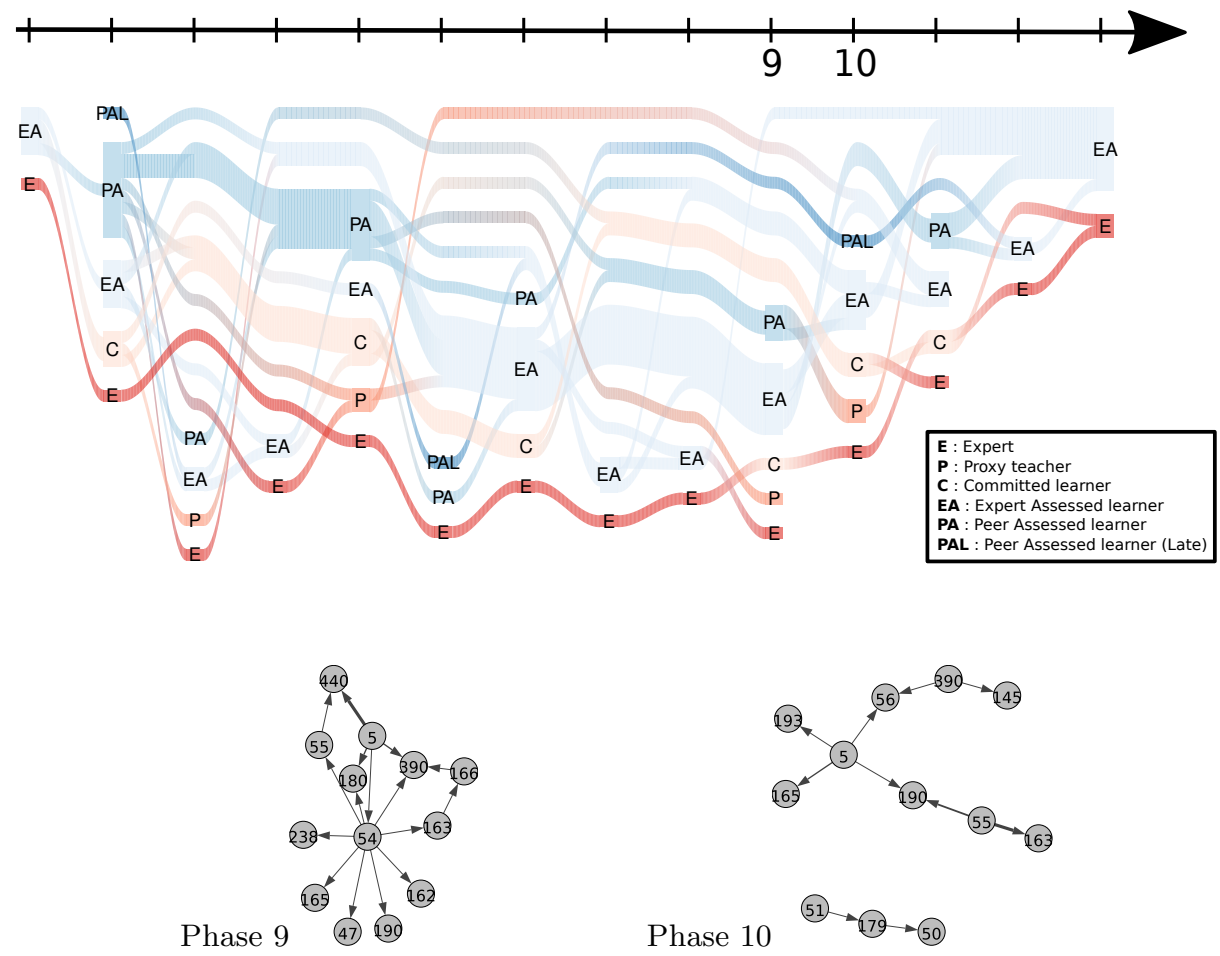

Fig. 2. The Sankey diagram representing the evolution of roles through the 14 phases of the course and the networks representing the validations that occurred during phases 9 and 10. Edge width depicts the number of validations (not taken into account in our measures).

Phase 10 arrives 35 days after phase 9 and lasts 11 days. As the teacher got a central position, s/he also occupies the Expert role again. Meanwhile, phase 9's very active user 54 has disappeared. Two students, 55 and 390, act as Proxy Teachers since they appear in the central position of star and in the co-assessor's position. Note that between the two phases, most of the students that were considered as Peer Assessed Learners have moved to Proxy Teachers, and are then validating their classmates.

\subsection{Overall role evolution}

In a complementary way, we finally consider the overall transitions between roles. For each user $u$, we filter the snapshots $G_{t}$ where $u$ is active. From the roles occupied by $u$ in each of these graphs, we get a sequence of roles. For each user, we can thus compute the number of times he or she passes from a role to another.

The overall role transition, depicted in Figure 3. A first remark is that most of the roles are somehow flexible even though most of the students seem to os- 


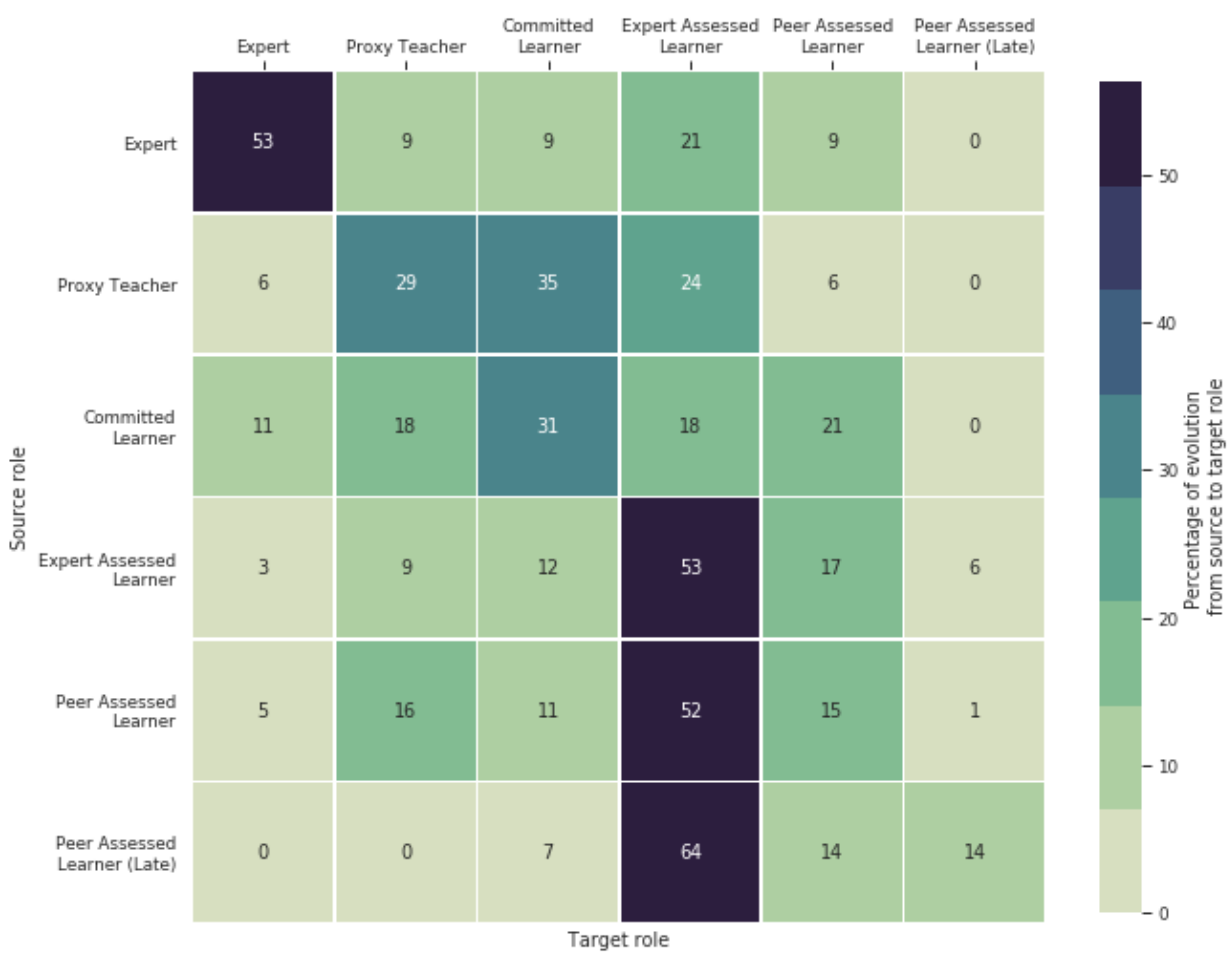

Fig. 3. Percentage of evolution from one role to another

cillate between roles Expert Assessed Learner and Peer Assessed Learner, which mainly differ from their propensity to be validated by the teacher. Note however that these roles also partly lead to the more active ones-Proxy Teacher and Committed Learner. Thus following the evolution of these roles could be a deeper insight.

Let us notice that the role Expert Assessed Learner seems to be a sink for other student roles: more than 50\% of the Expert Assessed Learners (Late or not) and the Peer Assessed ones become Expert Assessed Learners in the next phase. In the meanwhile, as said before, this role is not trapping the students in an posture of being evaluated by a teacher and leads also to assessor's positions. Its central position in the transition table invites for further investigations.

Concerning the assessors, it is notable that the occupiers of roles Expert and Committed Learners flow to every other roles except for the Expert Assessed Learner (Late) who are the last to validate their skills. Here it could be interesting to separate the students from the teachers in order to see if the latter ones are even more strictly confined to assessor's positions (and which ones), or conversely if teachers sometimes become assesses. 


\section{Conclusion}

We have introduced a methodology to detect roles in temporal networks. We have proposed a general framework for the development of a position-based embedding of nodes in snapshots. Thus a sequence of embedding reflects the evolution of topological roles that nodes occupy at different stages of an interaction network. We have illustrated this methodology on a case study based on the learning analytics field and demonstrated the power of expressiveness of graphlets and position-based embeddings.

Our work opens a number of avenues for additional research. Within a course, the teacher may authorize the self-assessment of skills. Enumerating graphlets with loops would reveal the influence of self-evaluation on the peer review mechanism. Within a curriculum it could be interesting to observe the evolution of a student's behaviour, and especially to use graphlets for the early detection of drop-out risk, which is a particularly important subject in the field of learning analytics that we have not yet addressed.

Another follow-up is the use of another kind of graphlets to track role through snapshots in order to compare the results. These graphlets could be size- 4 graphlets, others graphlets from literature [14] or disconnected graphlets [25], for instance. Another possible approach would be to focus on position transitions to characterize the role of each user through time.

\section{References}

1. Araujo, M., Papadimitriou, S., Günnemann, S., Faloutsos, C., Basu, P., Swami, A., Papalexakis, E.E., Koutra, D.: Com2: fast automatic discovery of temporal ('comet') communities. In: Pacific-Asia Conference on Knowledge Discovery and Data Mining. pp. 271-283. Springer (2014)

2. Authier, M., Lévy, P.: Les arbres de connaissances. La Découverte, Paris (1999), https://www.cairn.info/les-arbres-de-connaissances--9782707130440.htm

3. Braha, D., Bar-Yam, Y.: Time-dependent complex networks: Dynamic centrality, dynamic motifs, and cycles of social interactions. In: Adaptive Networks, pp. 39-50. Springer (2009)

4. Cela, K.L., Sicilia, M.Á., Sánchez, S.: Social network analysis in e-learning environments: A preliminary systematic review. Educational Psychology Review 27(1), 219-246 (2015)

5. Charbey, R., Prieur, C.: Stars, holes, or paths across your facebook friends: A graphlet-based characterization of many networks. Network Science pp. 1-22 (2019)

6. Dalsgaard, C.: Social software: E-learning beyond learning management systems. European Journal of Open, Distance and e-learning 9(2) (2006)

7. Dillenbourg, P.: What do you mean by collaborative learning? (1999)

8. Dunlavy, D.M., Kolda, T.G., Acar, E.: Temporal link prediction using matrix and tensor factorizations. ACM Transactions on Knowledge Discovery from Data (TKDD) 5(2), 10 (2011)

9. Ferguson, R., Shum, S.B.: Social learning analytics: five approaches. In: Proceedings of the 2nd international conference on learning analytics and knowledge. pp. 23-33. ACM (2012) 
10. Gartner, A., Kohler, M., Riessman, F., Grosjean, M.: Des enfants enseignent aux enfants: apprendre en enseignant. Hommes et groupes, Epi (1973), https://books. google.fr/books?id=y8DuPAAACAAJ

11. Gu, S., Milenkovic, T.: Graphlets versus node2vec and struc2vec in the task of network alignment. arXiv preprint arXiv:1805.04222 (2018)

12. Holland, P.W., Leinhardt, S.: Local structure in social networks. Sociological methodology 7, 1-45 (1976)

13. Holme, P., Saramäki, J.: Temporal networks. Physics reports 519(3), 97-125 (2012)

14. Hulovatyy, Y., Chen, H., Milenković, T.: Exploring the structure and function of temporal networks with dynamic graphlets. Bioinformatics 31(12), i171-i180 (2015)

15. Komarek, A., Pavlik, J., Sobeslav, V.: Network visualization survey. In: Computational Collective Intelligence, pp. 275-284. Springer (2015)

16. Kovanen, L., Karsai, M., Kaski, K., Kertész, J., Saramäki, J.: Temporal motifs in time-dependent networks. Journal of Statistical Mechanics: Theory and Experiment 2011(11), P11005 (2011)

17. Lloyd, S.: Least squares quantization in pcm. IEEE transactions on information theory 28(2), 129-137 (1982)

18. MacQueen, J., et al.: Some methods for classification and analysis of multivariate observations. In: Proceedings of the fifth Berkeley symposium on mathematical statistics and probability. vol. 1, pp. 281-297. Oakland, CA, USA (1967)

19. Milenković, T., Pržulj, N.: Uncovering biological network function via graphlet degree signatures. Cancer informatics 6, CIN-S680 (2008)

20. Milo, R., Shen-Orr, S., Itzkovitz, S., Kashtan, N., Chklovskii, D., Alon, U.: Network motifs: simple building blocks of complex networks. Science 298(5594), 824-827 (2002)

21. Paranjape, A., Benson, A.R., Leskovec, J.: Motifs in temporal networks. In: Proceedings of the Tenth ACM International Conference on Web Search and Data Mining. pp. 601-610. ACM (2017)

22. Paredes, W.C., Chung, K.S.K.: Modelling learning \&\#38; performance: A social networks perspective. In: Proceedings of the $2 \mathrm{Nd}$ International Conference on Learning Analytics and Knowledge. pp. 34-42. LAK '12, ACM, New York, NY, USA (2012), http://doi.acm.org/10.1145/2330601.2330617

23. Pržulj, N., Corneil, D.G., Jurisica, I.: Modeling interactome: scale-free or geometric? Bioinformatics 20(18), 3508-3515 (2004)

24. Rousseeuw, P.J.: Silhouettes: a graphical aid to the interpretation and validation of cluster analysis. Journal of computational and applied mathematics 20, 53-65 (1987)

25. Shervashidze, N., Vishwanathan, S., Petri, T., Mehlhorn, K., Borgwardt, K.: Efficient graphlet kernels for large graph comparison. In: Artificial Intelligence and Statistics. pp. 488-495 (2009)

26. Suh, H., Kang, M., Moon, K., Jang, H.: Identifying peer interaction patterns and related variables in community-based learning. In: Proceedings of th 2005 Conference on Computer Support for Collaborative Learning: Learning 2005: The Next 10 Years! pp. 657-661. CSCL '05, International Society of the Learning Sciences (2005), http://dl.acm.org/citation.cfm?id=1149293.1149379

27. Teplovs, C., Fujita, N., Vatrapu, R.: Generating predictive models of learner community dynamics. In: Proceedings of the 1st International Conference on Learning Analytics and Knowledge. pp. 147-152. LAK '11, ACM (2011)

28. Wernicke, S., Rasche, F.: Fanmod: a tool for fast network motif detection. Bioinformatics 22(9), 1152-1153 (2006) 